\title{
Metastatic Breast Cancer to the Bladder
}

\author{
Bijan Salari ${ }^{1,}{ }^{a}$ Daniel Rospert ${ }^{a},{ }^{b}$ Emmett Boyle ${ }^{c}$ Puneet Sindhwani (D) $a$ and Gregor Emmert ${ }^{c}$
}

Coresponding author(s): bijan.salari@utoledo.edu

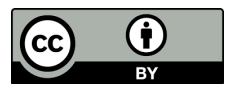

\begin{abstract}
${ }^{a}$ Department of Urology, The University of Toledo, Health Science Campus, 3000 Arlington Ave., Toledo, OH 43614, USA, ${ }^{b}$ M.D. Candidate, Class of 2022, The University of Toledo Heath Science Campus, 3000 Arlington Ave., Toledo, OH 43614, USA, and ${ }^{c}$ Promedica Genitourinary Surgeons, 2120 W. Central Ave Toledo, OH 43606, USA.
\end{abstract}

\begin{abstract}
Metastatic breast cancer to the bladder is extraordinarily rare, with only 66 cases reported in the literature to date. Occasionally, metastatic breast cancer can present itself with obstructive uropathy from a proposed retroperitoneal spread. We present a case of a 77 year old Caucasian female who was found to have acute urinary obstruction from metastatic breast cancer to the bladder. Her breast biopsy revealed invasive carcinoma with lobular features (Estrogen Receptor negative, Progesterone Receptor negative, and HER2 negative). She had symptoms of spontaneous urinary incontinence, but no gross hematuria. She had elevated Chromogranin A, Cancer Antigen (CA) 19-9, and Carinoembryonic antigen (CEA). She had a bladder biopsy which demonstrated metastatic breast carcinoma that stained GATA3, GCDFP15, BerEp4 positive and CK20 negative. $0 \%$ of tumor cells were positive for Programmed death-ligand (PD-L) 1 expression. The patient had renal deterioration despite bilateral ureteral stent placement, and thus required percutaneous nephrostomy tube placement. She died 4 months after her initial diagnosis.
\end{abstract}

breast cancer | metastasis | bladder cancer

$\mathbf{M}$ etastatic breast cancer to the bladder is extraordinarily rare, with only 66 cases reported in the literature to date. Occasionally, metastatic breast cancer can present itself with obstructive uropathy from a proposed retroperitoneal spread. We present a case of a 77 year old Caucasian female who was found to have acute urinary obstruction from metastatic breast cancer to the bladder. Her breast biopsy revealed invasive carcinoma with lobular features (Estrogen Receptor negative, Progesterone Receptor negative, and HER2 negative). She had symptoms of spontaneous urinary incontinence, but no gross hematuria. She had elevated Chromogranin A, Cancer Antigen (CA) 19-9, and Carinoembryonic antigen (CEA). She had a bladder biopsy which demonstrated metastatic breast carcinoma that stained GATA3, GCDFP15, BerEp4 positive and CK20 negative. 0\% of tumor cells were positive for Programmed deathligand (PD-L) 1 expression. The patient had renal deterioration despite bilateral ureteral stent placement, and thus required percutaneous nephrostomy tube placement. She died 4 months after her initial diagnosis.

\section{Case Report}

\section{Patient Information}

We present a 77 year old Caucasian female with no significant past medical history who was found to have metastatic breast cancer to the bladder, causing obstructive uropathy.

\section{Objective for Case Reporting}

Because metastatic breast cancer to the bladder is so uncommon, our goal is to contribute to scarce literature on the presenting signs, symptoms, and pathology associated with this disease process.

Case. We present a 77 year old female who initially presented to primary care with back pain and hip pain that radiated down her legs, as well as spontaneous urinary incontinence for 1 month, which was not associated with urgency or increased abdominal pressure. She did not have gross hematuria. Urinalysis with microscopy showed 2 red blood cells (RBC), 3 white blood cells (WBC), and 1 squamous epithelial cell per high power field (HPF). Urine culture was no growth. Magnetic Resonance Imaging (MRI) of her lumbar spine with and without contrast demonstrated diffuse osseus metastatic lesions of the pelvis, thoracic and lumbar spine; however, she had no lesions of the spinal cord.

Her yearly mammogram two months prior was negative for malignancy with a yearly follow up recommended. Her father's aunt had breast cancer at age 50. Further staging workup including a Computed Tomography (CT) of the chest with contrast demon-

Submitted: 08/14/2019, published: 05/05/2020 
strated a $2.5 \times 2.4 \times 2.2 \mathrm{~cm}$ inferior right breast soft tissue density. CT abdomen and pelvis with contrast demonstrated bilateral hydroureteronephrosis (Figure 1), irregularity of the duodenum and pancreatic head, and diffuse osseus disease as previously noted. There was no irregularity of the bladder. Chromogranin A was mildly elevated at $103 \mathrm{ng} / \mathrm{mL}$ (normal $<98 \mathrm{ng} / \mathrm{mL}$ ), Cancer Antigen (CA) 19-9 was elevated at 9,572 U/mL (normal $<35 \mathrm{U} / \mathrm{mL}$ ), and Carcinoembryonic antigen (CEA) was elevated at 182 (normal $<5 \mathrm{ng} / \mathrm{mL}$ ). She underwent a breast biopsy with pathology demonstrating invasive carcinoma with lobular features (Estrogen Receptor negative, Progesterone Receptor negative, and HER2 negative) as well as left iliac bone fine needle aspirate and core biopsy showed signet ring morphology consistent with origin from lobular breast carcinoma. Patient also underwent endoscopic ultrasound guided biopsies of the pancreas and duodenum which were negative for malignancy.

Because the patient had bilateral hydroureteronephrosis, urology urgently took the patient to the operating room for cystoscopy, bladder biopsy, and bilateral ureteral stent placement to preserve kidney function for potential future chemotherapy. Serum creatinine was 1.28 at time of admission. Cystoscopy demonstrated an irregular bladder mass on the right and left lateral wall of the bladder (Figure 2).

Biopsy demonstrated metastatic breast carcinoma that stained GATA3, GCDFP15, BerEp4 positive and CK20 negative. 0\% of tumor cells were positive for Programmed death-ligand (PD-L) 1 expression. Despite bilateral ureteral stent placement, the patient's kidney function deteriorated and she required bilateral percutaneous nephrostomy tube placement three days post-operatively to maximize urinary drainage. Patient was discharged with plans for palliative chemotherapy, however was re-admitted with persistent GI symptoms of nausea and vomiting and jaundice. She was discharged to hospice and died approximately 4 months after her initial diagnosis.

\section{Discussion}

Breast cancer is the most diagnosed cancer, as well as the leading cause of cancer death among women worldwide (1). Metastatic breast cancer to the bladder is exceedingly rare. To date, 66 cases have been reported in the literature and patients most often present with painless gross hematuria, and in very rare cases renal failure from obstructive uropathy. Our patient did have urinary symptoms of spontaneous urinary incontinence with low post void residuals, however she had no evidence of microscopic or gross hematuria. Her initial presentation included acute kidney injury with obstructive uropathy, possibly from retroperitoneal extension. In agreement with the literature review, our case demonstrated lobular, rather than ductal, carcinoma of the breast to metastasize to the genitourinary system (2). The tumor demonstrated no PD-L1 expression, which has been implicated as a target for other bladder cancer therapies. The patient was concomitantly diagnosed with both breast and bladder carcinoma, in agreement with prior literature (3). As was the case for most prior reports, the patient's tumor was a highly aggressive Itriple negative" carcinoma with a short length of survival only four months after diagnosis (4). As more cases our reported, we can better define the pathology, clinical symptoms and signs, and appearance of bladder cancer as a result of breast metastasis.

\section{Conclusion}

Metastatic breast cancer to the bladder is exceedingly rare, but can first manifest itself as acute urinary obstruction. The prognosis of a patient with this condition is poor, with life expectancy after diagnosis typically less than 1 year.

\section{Conflict of interest}

Authors declare no conflict of interest.

\section{Authors' contributions}

BS wrote and edited manuscript, DR literature review, EB, PS, GE reviewed paper. All authors read and approved the final document.

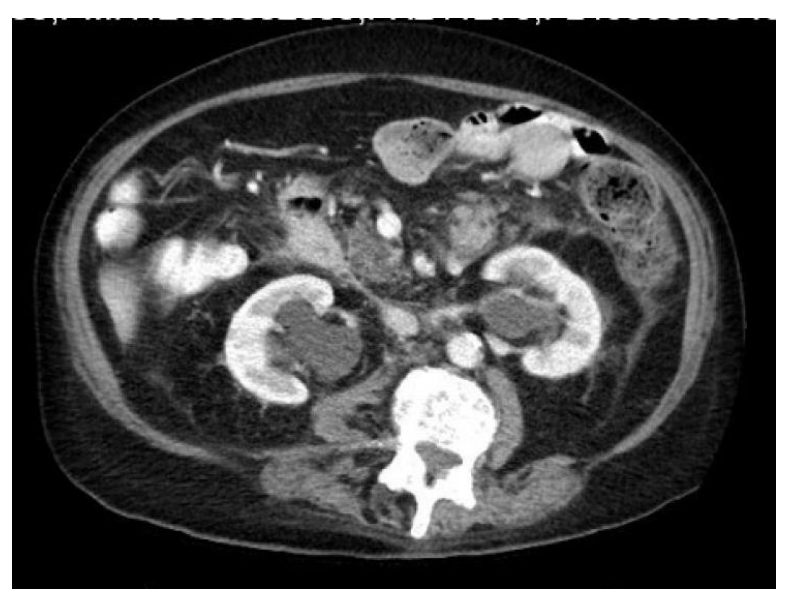

Figure 1. CT abdomen and pelvis with contrast showing bilateral hydronephrosis.

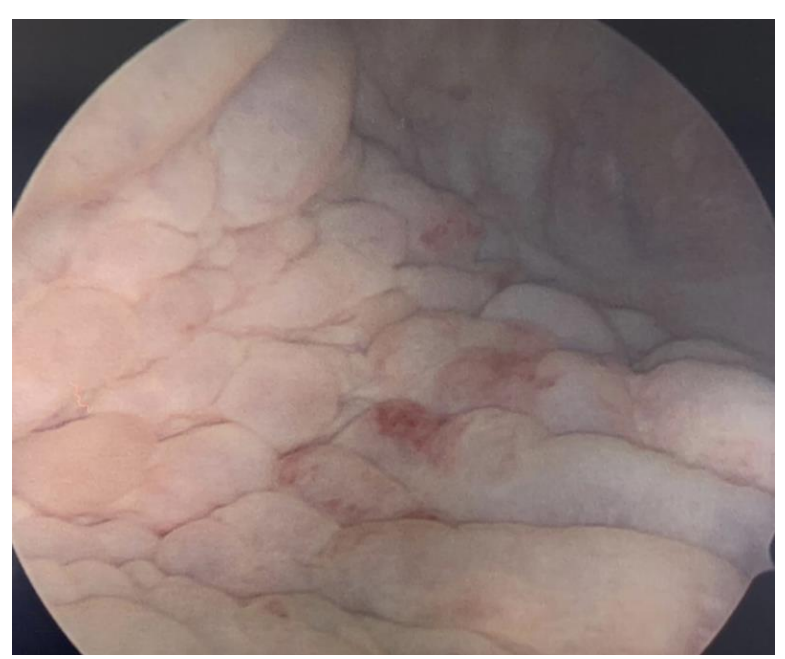

Figure 2. Irregular right lateral wall bladder mucosa at the time of cystoscopy. 
1. LA, Bray F, Siegel RL, et al. (2015) Global cancer statistics, 2012. CA Cancer J Clin. 65(2):87-108.

2. MJ, Ingold JA. (1993) Metastatic patterns of invasive lobular versus invasive ductal carcinoma of the breast. Surgery 114(4):637-641.
3. Y, Opsomer R, Donnez J, et al. (1982) Bladder metastases from breast cancer: 2 cases. Acta Urol Belg. 50(1):87-90.

4. Hagemeister FB, Buzdar AU, Luna MA, et al. (1980) Causes of death in breast cancer: a clinicopathologic study. Cancer 46(1):162-167. 